\title{
An Extension of the SSG Model on Compressible Turbulent Flow
}

\author{
K. Hechmi ${ }^{\dagger}$ and L. Taieb \\ Department of Physics, Faculty of Sciences, Tunis, Elmanar, 1060, Tunisia \\ †Corresponding Author Email: khlifihachmi@yahoo.fr \\ (Received August 28, 2010; accepted May 14, 2011)
}

\begin{abstract}
This work focuses on the performance and validation of some recent Reynolds stress models in compressible homogeneous shear flow. The SSG model developed by Speziale Sarkar and Gatski has shown a great success in simulating a variety of incompressible complex turbulent flows. On the other hand, it has not predicted correctly the compressible turbulence at high speed shear flow. Thus, a compressibility correction for this model is the major aim of this study. In the present work, two recent compressible models for the pressure strain-strain correlation have been used to modify the linear term of the SSG model. These modifications make the linear term dependent on a turbulent Mach number. In addition, compressibility correction model for the slow part of the pressure strain is proposed. The obtained results are compared with DNS results of Sarkar. The results show that important parameters characteristic of compressibility in homogeneous turbulent shear flow are well captured by the extended SSG model.
\end{abstract}

Keywords: Compressible, Turbulence, Pressure-Strain, Models of Turbulence, Shear flow.

\section{NOMENCLATURE}

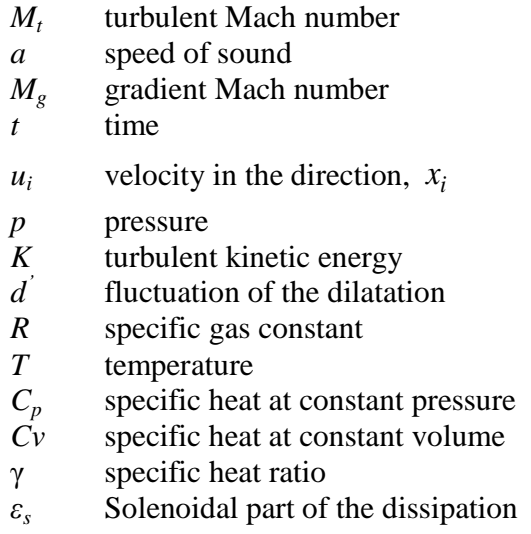

\section{INTRODUCTION}

Several experiments and numerical simulations studies have been performed during the last decade to understand the compressibility effects on the turbulence. Compressible turbulent flows arise in many engineering applications related to the combustion, environment, spacecraft, and hypersonic flight problems. The work recently developed by Younis et al. (2009) is one of these types of research in which computational and

$\begin{array}{ll}\mu & \text { viscosity coefficient } \\ \rho & \text { density } \\ \varepsilon & \text { dissipation rate of turbulence } \\ \delta_{i j} & \text { Kronecker delta } \\ \kappa & \text { thermal conductivity } \\ \varepsilon_{C} & \text { dilatational part of the dissipation } \\ b_{i j} & \text { Reynolds stress anisotropy } \\ R_{i j,} & \text { Reynolds stress intensity } \\ ()^{,} & \text {Favre fluctuation } \\ ()^{\prime} & \text { Reynolds fluctuation } \\ \overline{()} & \text { standard ensemble mean } \\ \tilde{()} & \text { Favre average } \\ ()_{i} & \text { spatial gradient }\end{array}$

analysis of the supersonic turbulent flow in complex configuration are examined. In particular, compressible homogeneous shear flow is mainly an important motivation for some authors. The reason behind that motivation is that compressible homogeneous shear flow summarizes some of the important of flow compressibility effects in a simplified setting in which the complex effects of turbulent diffusion are not considered. It has been a useful test case for the calibration and testing of variety of turbulence models. In this context, many 
studies of the compressible shear flow show that the changes of the turbulence structures are principally due to the structural compressibility effects which significantly affect the pressure field and then the pressure-strain correlation. Eventually, the pressurestrain correlation appears as the main factor for the changes in the magnitude of the Reynolds stress anisotropies. The extension of the standard models to compressible flows represents a research topic of great scientific and industrial interest. A major challenge related to this extension is to take into account the compressibility effects in the classical scheme closures of turbulence. In this context, extensive research initiatives in compressible modeling have been taken during the last few years. It is well concluded that the Favre Reynolds stress closure using the standard models of the pressure strain correlation with the addition of the compressible dissipation and pressure-dilatation correlation models failed to predict high compressible flows (see Speziale et al. 1995). Sarkar (1995) and Fujihiro Hamba (1999) also performed DNS results of compressible homogeneous shear flow and reached similar conclusions concerning the roles of dilatational terms. They found out a notable decrease of the growth rate of the turbulent kinetic energy when the values of the turbulent Mach number increase, the reduction of the turbulence levels arising from compressibility effects is related to the inhibited turbulence production and not the explicit dilatational terms. These conclusions are confirmed by Vreman et al. (1996) and Pantano et al. (2003) in their DNS results which show that compressibility terms do not affect the compressible mixing layer. In contrast to dilatational effects, the structural compressibility effects strongly affect the pressure field, so the pressure fluctuations were reduced. The consequent effects on the pressurestrain correlation may cause significant changes on turbulence structures. According to the DNS results of Sarkar (1995), there is a reduction in the magnitude of the Reynolds shear stress anisotropy and an increase in the magnitude of the normal stress anisotropy. As a consequence, the pressure strain modeling seems to be an important issue in the second order closures for the compressible turbulent flows.

An extension of the non-linear incompressible model of Speziale et al. (1991) to compressible shear flows is the major aim of the present work. In the present work, two linear models due to Adumitroiae et al. (1999) and Park et al. (2005) are used to make the linear terms of the SSG model dependent on an extra compressibility parameter: the turbulent Mach number $M_{t}\left(M_{t}=\sqrt{2 K} / \bar{a}\right)$, where $\bar{a}$ is the mean speed of sound). The validity of the proposed compressibility corrections of the SSG model has been tested for four selected cases from the DNS results of Sarkar (1995) for compressible homogeneous shear flow.

\section{GOVERNING EQUATIONS}

The general equations governing the motion of a compressible fluid are the Navier Stokes equations.
They can be written as follows for mass, momentum and energy conservation:

$$
\begin{aligned}
& \frac{\partial}{\partial t} \rho+\frac{\partial}{\partial x_{i}} \rho u_{i}=0 \\
& \frac{\partial}{\partial t} \rho u_{i}+\frac{\partial}{\partial x_{j}} \rho u_{i} u_{j}=\frac{\partial}{\partial x_{j}} \sigma_{i j} \\
& \frac{\partial}{\partial t} \rho e+\frac{\partial}{\partial x_{j}} \rho e u_{j}=\frac{\partial}{\partial \mathrm{x}_{\mathrm{j}}} \sigma_{i j} u_{i}-\frac{\partial}{\partial x_{j}}\left(\kappa T_{, j}\right)
\end{aligned}
$$

Where $\quad e=c_{v} T, \tau_{i j}=2 \mu\left(u_{i, j}+u_{j, i}\right)$ and $\sigma_{i j}=-p \delta_{i j}+\tau_{i j} \quad$ The Favre averaged equations are:

$$
\begin{gathered}
\frac{\partial}{\partial t} \bar{\rho}+\frac{\partial}{\partial x_{i}}\left(\bar{\rho} \tilde{U}_{i}\right)=0 \\
\frac{\partial}{\partial t}\left(\bar{\rho} \tilde{U}_{i}\right)+\frac{\partial}{\partial x_{i}}\left(\bar{\rho} \tilde{U}_{i} \tilde{U}_{j}\right)= \\
\frac{\partial}{\partial x_{j}}\left(\tilde{\sigma}_{i j}+\overline{\tau_{i j}^{\prime \prime}}-\frac{\partial}{\partial x_{j}} \overline{\rho u_{i}^{\prime \prime} u_{j}^{\prime \prime}}\right) \\
\frac{\partial}{\partial t} \bar{\rho} \tilde{e}+\frac{\partial}{\partial x_{j}} \bar{\rho} \tilde{e} \tilde{U}_{j}=-\varphi_{\mathrm{e}} \\
+\pi_{d}-\frac{\partial}{\partial \mathrm{x}_{\mathrm{j}}} c_{v} \overline{\rho u_{i}^{\prime \prime} T^{\prime \prime}}
\end{gathered}
$$

where

$$
\begin{aligned}
& \left.\varphi_{e}=\bar{p} \frac{\partial}{\partial x_{i}}\left(\tilde{U}_{i}+\overline{u_{i}^{\prime \prime}}\right)+\frac{\partial}{\partial x_{i}} \overline{\left(\kappa \frac{\partial}{\partial x_{i}} T\right.}\right)+\overline{\tau_{i j} u_{i, j}} \\
& \tilde{\tau}_{i j}=2 \mu \tilde{S}_{i j}-\frac{2}{3} \bar{\mu} \tilde{U}_{k, k} \delta_{i j} \\
& \tilde{S}_{i j}=\left(\tilde{U}_{i, j}-\tilde{U}_{j, i}\right) / 2 \\
& \pi_{d}=\overline{p^{\prime} d^{\prime}}=\overline{p^{\prime} u_{i, i}^{\prime}}
\end{aligned}
$$

The Favre averaged Reynolds stress $R_{i j}=\overline{\rho u_{i}^{\prime \prime} u_{j}^{\prime \prime}} / \bar{\rho}$ are solutions of the transport equation, namely

$$
\begin{array}{r}
\frac{\partial}{\partial t}\left(\bar{\rho} R_{i j}\right)+\frac{\partial}{\partial x_{m}}\left(\bar{\rho} \tilde{U}_{m} R_{i j}\right)=P_{i j}+ \\
D_{i j}+\phi_{i j}+\varepsilon_{i j}+V_{i j}
\end{array}
$$

where the symbols $P_{i j}, D_{i j m}, \phi_{i j}, \varepsilon_{i j}$ and $V_{i j}$ represent turbulent production, turbulent diffusion, pressure strain correlation, turbulent dissipation and the mass flux variation respectively.

$$
\begin{aligned}
& P_{i j}=-\bar{\rho} R_{j m} \tilde{U}_{i, m}-\bar{\rho} R_{i m} \tilde{U}_{j, m} \\
& \overline{D_{i j m}}=-\left(\overline{\rho u_{i}^{\prime \prime} u_{j}^{\prime \prime} u_{m}^{\prime \prime}}+\overline{p^{\prime} u_{j}^{\prime \prime}} \delta_{i m}+\overline{p^{\prime} u_{i}^{\prime \prime}} \delta_{j m}-\right. \\
& \left.\overline{\tau_{i m}^{\prime \prime} u_{j}^{\prime \prime}}-\overline{\tau_{j m}^{\prime \prime} u_{i}^{\prime \prime}}\right) \\
& \phi_{i j}=\overline{p^{\prime}\left(u_{i, j}^{\prime \prime}+u_{j, i}^{\prime \prime}\right)}=\phi_{i j}^{*}+\frac{2}{3} \overline{p^{\prime} u_{k, k}^{\prime \prime}} \delta_{i j}
\end{aligned}
$$




$$
\begin{aligned}
& \varepsilon_{i j}=\overline{\tau_{i m}^{\prime \prime} u_{j, m}^{\prime \prime}}-\overline{\tau_{j m}^{\prime \prime} u_{i, m}^{\prime \prime}} \\
& V_{i j}=-\bar{p}_{, j} \overline{u_{i}^{\prime \prime}}+-\bar{p}_{, i} \overline{u_{j}^{\prime \prime}}+\tilde{\tau}_{i m, m} \overline{u_{j}^{\prime \prime}}+ \\
& \quad \tilde{\tau}_{j m, m} \overline{u_{i}^{\prime \prime}}
\end{aligned}
$$

Classically, the second order closure suggests to determine the dissipation term $\varepsilon_{i j}$ by using isotropic dissipation model:

$\varepsilon_{i j}=\frac{2}{3} \varepsilon \delta_{i j}$

Recently a concept of the dissipation in compressible turbulence was proposed by Sarkar (1991), Zeman (1990) as

$$
\varepsilon=\varepsilon_{s}+\varepsilon_{c}
$$

where, for homogeneous shear flow turbulence $\bar{\rho} \varepsilon_{s}=\overline{\mu \omega_{i}^{\prime} \omega_{i}^{\prime}}, \omega_{i}^{\prime}$ is the fluctuating vorticity, and $\varepsilon_{c}=\frac{4}{3} \overline{\mu u_{k, k}^{\prime 2}}$. The authors argued that the solenoidal part of the dissipation can be modeled by using the traditional incompressible equation model, namely:

$$
\begin{array}{r}
\frac{\partial}{\partial t}\left(\bar{\rho} \varepsilon_{s}\right)+\frac{\partial}{\partial x_{k}}\left(\bar{\rho} \varepsilon_{s} \tilde{U}_{k}\right)=\bar{\rho} \frac{\varepsilon_{s}}{K} \\
\left(C_{\varepsilon 1} R_{k m} \frac{\partial}{\partial x_{m}} \tilde{U}_{k}-C_{\varepsilon 2} \varepsilon_{s}\right)- \\
\frac{\partial}{\partial x_{k}}\left(C_{\varepsilon 3} \bar{\rho} \frac{K}{\varepsilon_{s}} R_{k m} \frac{\partial}{\partial x_{m}} \varepsilon_{s}\right)
\end{array}
$$

The compressible dissipation $\varepsilon_{c}$ is determined by the commonalty used models as

$$
\varepsilon_{c}=g_{c} \varepsilon_{s}
$$

$g_{c}$ is a function of the turbulent Mach number.

\section{Compressible TurbulenCe Models For THE PRESSURE Strain CORRELATION}

Many DNS and experiment results have been carried out on compressible turbulent flows, most of which show the significant compressibility effects on the pressure-strain correlation via the pressure field. Such effects induce reduction in the magnitude of the anisotropy of the Reynolds shear stress and increase in the magnitude of the normal stress anisotropy. Consequently, the pressure-strain correlation requires a careful modeling in the Reynolds stress turbulence model. With respect to the incompressible case, many compressible models have been developed for the pressure-strain correlation. Hereafter, most of all these models are generated from a simple extension of its incompressible counter-part. In general, they perform well in the simulation of important turbulent flows evolving with moderate compressibility.

\subsection{Model of Adumitroiae et al. (1999)}

Adumitroiae et al. assumed that incompressible modeling approach of the pressure-strain can be used to develop turbulent models taking into account compressibility effects. Considering a non zero divergence for the velocity fluctuations called the compressibility continuity constraint and using different models for the pressure dilatation which is proportional to the trace of the pressure-strain, their model for the pressure strain is written as follows:

$$
\begin{aligned}
& \phi_{i j}^{*}=-C_{1} \bar{\rho} \varepsilon_{s} b_{i j}+\left(\frac{4}{5}+\frac{2}{5} d_{1}\right) \\
& \bar{\rho} \mathrm{K}\left(\tilde{S_{i j}}-\frac{1}{3} \tilde{S}_{l l} \delta_{i j}\right)+2 \bar{\rho} K \\
& \left(1-C_{3}+2 d_{2}\right)\left[b_{i k} \tilde{S_{j k}}+b_{j k} \tilde{S_{i k}}\right. \\
& \left.-\frac{2}{3} b_{m l}{\tilde{S_{m l}}}_{i j}\right]-\bar{\rho} K\left(1-C_{4}-2 d_{2}\right) \\
& {\left[b_{i k} \tilde{\Omega}_{j k}+b_{j k} \tilde{\Omega}_{i k}-\frac{4}{3} d_{2} \tilde{S_{k k}} b_{i j}\right]}
\end{aligned}
$$

where

$\tilde{S}_{i j}=0.5\left(\tilde{U}_{i, j}+\tilde{U}_{j, i}\right), \tilde{\Omega}_{i j}=0.5\left(\tilde{U}_{i, j}-\tilde{U}_{j, i}\right)$ and

$b_{i j}=R_{i j} / 2 K-\frac{1}{3} \delta_{i j}$

The compressible coefficients $d_{1}$ and $d_{2}$ are determined from some compressible closures for the pressure-dilatation correlation (see Adumitroiae et al. 1999).

\subsection{Model of Park et al. (2005)}

The authors used the concept of moving equilibrium in homogeneous shear flow to modify the linear pressure strain term part as follows:

$$
\begin{aligned}
& \phi_{i j}^{*}=-C_{1} \bar{\rho} \varepsilon_{s} b_{i j}-\left(B_{4}+1.2 F\left(M_{t}\right)\right) \\
& \bar{\rho} \mathrm{K} \tilde{S}_{i j}^{*}-\left(B_{2}+\alpha F\left(M_{t}\right)\right. \\
& \bar{\rho} K\left(P_{i j}-\frac{2}{3} P_{k} \delta_{i j}\right)-\left(B_{3}+F\left(M_{t}\right)\right. \\
& \bar{\rho} K\left(D_{i j}-\frac{2}{3} P_{k} \delta_{i j}\right)-B_{5} b_{i j} \tilde{S}_{k k}
\end{aligned}
$$

where

$F\left(M_{t}\right)=F_{\max }\left[1-\exp \left(-\left(\beta M_{t}\right)^{2}\right)\right]$

$F_{\max }=\frac{B_{2}-2 B_{3}}{2+\alpha}$

$P_{i j}=-\bar{\rho} R_{j m} \tilde{U}_{i, m}-\bar{\rho} R_{i m} \tilde{U}_{j, m}$

$D_{i j}=-\bar{\rho} R_{j m} \tilde{U}_{m, i}-\bar{\rho} R_{i m} \tilde{U}_{m, j}$

For the function $F\left(M_{t}\right), \beta=4$ and $\alpha$ between 1 and 4 are the suited values by Park et al. One can see that, when $F\left(M_{t}\right)=0$, the model like the original Launder Reece and Rodi (LRR) model (1975) and the coefficients $C_{1}, B_{2}, B_{3}, B_{4}$ and $B_{5}$ are: 
$B_{2}=\frac{8+C}{11}, B_{3}=\frac{8 C-2}{11}$

$B_{4}=\frac{60 C-4}{55}, B_{5}=\frac{6 C+4}{11}$

0 and $C=0.4$

\section{A Priori an Extension of the SSG MODEL}

Speziale Sarkar and Gatski (1991) developed a model for the pressure-strain correlation namely:

$\phi_{i j}^{*}=-\left(C_{1} \bar{\rho} \varepsilon_{s}+C_{1}^{*} \bar{\rho} P\right) b_{i j}$

$+\left(C_{3}-C_{3}^{*} I I^{1 / 2}\right) \bar{\rho} \mathrm{K}\left(\tilde{S_{i j}}-\frac{1}{3} \tilde{S_{l l}} \delta_{i_{j}}\right)$

$+C_{2} \bar{\rho} \varepsilon_{s}\left(b_{i k} b_{k j}-\frac{1}{3} b_{m k} b_{k m} \delta_{i j}\right)$

$+\bar{\rho} K C_{4}\left[b_{i k} \tilde{S}_{j k}+b_{j k} \tilde{S_{i k}}-\frac{2}{3} b_{m l} \tilde{S_{m l}} \delta_{i j}\right]$

$+\bar{\rho} K C_{5}\left[b_{i k} \tilde{\Omega}_{j k}+b_{j k} \tilde{\Omega}_{i k}-\frac{4}{3} d_{2} \tilde{S_{k k}} b_{i j}\right.$

where $C_{1}, C_{1}^{*}, C_{2}, C_{3}, C_{4}$ and $C_{5}$ are:

$C_{1}=3.4, C_{1}^{*}=1.8, C_{2}=4.2, C_{3}=0.8$

$C_{4}=1.25, \quad C_{5}=0.4$.

$I I=b_{i j} b_{i j}$ and $P=-R_{i j} \tilde{U}_{i, j}$.

The pressure strain $\left(\phi_{i j}^{*}\right)_{S S G}$ can be split in two parts: $\left(\phi_{i j}^{*}\right)_{l i n}$ is linear of the mean strain and Reynolds stress which uses a formulation like that of Launder Reece and Rodi (1975). Moreover, the non-linear part $\left(\phi_{i j}^{*}\right)_{n o n-l i n}$ is quadratic in the Reynolds stress. Thus, we can write:

$\left(\phi_{i j}^{*}\right)_{S S G}=\left(\phi_{i j}^{*}\right)_{l i n}+\left(\phi_{i j}^{*}\right)_{n o n-l i n}$

where

$\left(\phi_{i j}^{*}\right)_{l i n}=-C_{1} \bar{\rho} \varepsilon_{s} b_{i j}+C_{3} \bar{\rho} \mathrm{K}\left(\tilde{S}_{i j}-\frac{1}{3} \tilde{S}_{l l} \delta_{i j}\right)$

$+\bar{\rho} K C_{4}\left[b_{i k} \tilde{S}_{j k}+b_{j k} \tilde{S}_{i k}-\frac{2}{3} b_{m l} \tilde{S}_{m l} \delta_{i j}\right]$

$+\bar{\rho} K C_{5}\left[b_{i k} \tilde{\Omega}_{j k}+b_{j k} \tilde{\Omega}_{i k}-\frac{4}{3} d_{2} \tilde{S}_{k k} b_{i j}\right.$

$\left(\phi_{i j}^{*}\right)_{n o n-l i n}=-C_{1}^{*} \bar{\rho} P b_{i j}-$

$C_{3}^{*} I I^{1 / 2} \bar{\rho} \mathrm{K}\left(\tilde{S_{i j}}-\frac{1}{3} \tilde{S_{l l}} \delta_{i_{j}}\right)$

$+C_{2} \bar{\rho} \varepsilon_{s}\left(b_{i k} b_{k j}-\frac{1}{3} b_{m k} b_{k m} \delta_{i j}\right)$

The starting point of the proposed compressibility modification of this model is from the several analysis and development in which Pope (1994) establishes correspondence between some second order closure models. Here, we concentrate on the modification of $\left(\phi_{i j}^{*}\right)_{l i n}$ and we propose to write: $\left(\phi_{i j}^{*}\right)_{S S G}=\left(\phi_{i j}^{*}\right)_{l i n}^{c o m p}+\left(\phi_{i j}^{*}\right)_{n o n-l i n}$

Different compressible models can be used for $\left(\phi_{i j}^{*}\right)_{\text {lin }}^{\text {comp }}$ which is essentially extension of the LRR model (1975) using different extra compressibility parameters like pressure variance, gradient Mach number and turbulent Mach number. Two compressible models due to Adumitroiae et al. (1999) and Park et al. (2005) are used to express $\left(\phi_{i j}^{*}\right)_{\text {lin }}^{\text {comp }}$. Thus, the SSG model becomes dependent on the turbulent Mach turbulent number:

$$
\begin{aligned}
& \left(\phi_{i j}^{*}\right)_{S S G}=\left(\left(\phi_{i j}^{*}\right)_{\text {lin }}^{\text {comp }}\right)^{\text {Adumitroiae }}+\left(\phi_{i j}^{*}\right)_{\text {non-lin }} \\
& \left(\phi_{i j}^{*}\right)_{S S G}=\left(\left(\phi_{i j}^{*}\right)_{\text {lin }}^{\text {comp }}\right)^{\text {Park }}+\left(\phi_{i j}^{*}\right)_{n o n-l i n}
\end{aligned}
$$

All the model constants are summarized in Table 1.

Table 1 Compressibility correction of the SSG

\begin{tabular}{|c|c|}
\hline \multirow{4}{*}{ SSGi } & $C_{1}=3.4$ \\
\hline & $C_{3}=0.8$ \\
\hline & $C_{4}=1.25$ \\
\hline & $C_{5}=0.4$ \\
\hline \multirow[b]{4}{*}{ SSGa } & $C_{1}=3.4$ \\
\hline & $C_{3}=0.8$ \\
\hline & $C_{4}=1.25+0.3 M_{t}$ \\
\hline & $C_{5}=0.4-0.3 M_{t}$ \\
\hline \multirow{4}{*}{ SSGp } & $C_{1}=3.4$ \\
\hline & $C_{3}=0.8+\lambda_{1} F$ \\
\hline & $C_{4}=1.25+\lambda_{2} F$ \\
\hline & $C_{5}=0.4+\lambda_{3} F$ \\
\hline
\end{tabular}
coefficients model of $\left(\phi_{i j}^{*}\right)_{\text {lin }}^{\text {comp }}$

$\lambda_{1}=\frac{4}{3}(1+\alpha)-\frac{6}{5}, \lambda_{2}=2(1+\alpha)$

$\lambda_{3}=2(\alpha-1)$

$F=\frac{0.54}{2+\alpha}\left(1-\exp \left(-\left(4 M_{t}\right)^{2}\right)\right)$

The SSGa and SSGp are the modified versions of the standard model SSGi by using Adumitroiae et al. (1999) and Park et al. (2005) respectively. 


\section{Modeling The Slow Part Of The Pressure-STRAIN CORRELATION}

The slow part of the pressure strain correlation describes the return to isotropy behavior of the turbulence. This behavior of the flow is observed when the mean velocity gradients are removed (see Park et al. 2005). Most models like Rotta (1951) use linear formulation:

$$
\phi_{i j s}^{*}=-C_{1} \bar{\rho} \varepsilon_{s} b_{i j}
$$

Now the purpose is to investigate compressibility effects in this term by modifying the standard coefficient $C_{1}$. Using the general formulation published by Pope (1994). $\phi_{i j s}$ is written as follow:

$$
\begin{aligned}
\phi_{i j s}= & \left(\frac{2}{3}+C_{0}\right) \varepsilon_{s} \delta_{i j}+ \\
& G_{i l}\left(R_{l j}\right)+G_{j l}\left(R_{l i}\right)
\end{aligned}
$$

with the tensor $G_{i j}$ given by

$$
G_{i j}=\frac{\varepsilon_{s}}{K}\left(\alpha_{1} \delta_{i j}+\alpha_{2} b_{i j}+\alpha_{3} b_{i j}^{2}\right)
$$

where $\alpha_{1}, \alpha_{2}, \alpha_{3}$ and $C_{0}$ are model constants. Here, a simplification which corresponds to the choice of $G_{i j}$ as an isotropic tensor is considered:

$G_{i j}=\frac{\varepsilon_{s}}{K} \alpha_{1} \delta_{i j}$

and we obtain

$$
\phi_{i j s}=\left(\frac{2}{3}+C_{0}+\frac{4}{3} \alpha_{1}\right) \varepsilon_{s} \delta_{i j}+4 \alpha_{1} \varepsilon_{s} b_{i j}
$$

The contraction $i=j$ in this equation leads to:

$$
\phi_{i i s} / \bar{\rho}=\left(2+3 C_{0}+4 \alpha_{1}\right) \varepsilon_{s}
$$

Because

$\phi_{i i s}=2\left(\overline{p^{\prime} d^{\prime}}\right)_{s}$

It comes

$$
\frac{\overline{\left(p^{\prime} d^{\prime}\right)_{s}} / \bar{\rho}}{2 \varepsilon_{s}}=\frac{1}{2}+\frac{3}{4} C_{0}+\alpha_{1}
$$

and the coefficient $\alpha_{1}$ is given by

$\alpha_{1}=\frac{\overline{\left(p^{\prime} d^{\prime}\right)_{s}} / \bar{\rho}}{2 \varepsilon_{s}}-\left(\frac{1}{2}+\frac{3}{4} C_{0}\right)$

For incompressible turbulence, $\left(\overline{p^{\prime} d^{\prime}}\right)_{s}$ vanishes and $\alpha_{1}^{\text {inc }}$ is given in Pope (1994) as

$$
\alpha_{1}^{i n c}=-\left(\frac{1}{2}+\frac{3}{4} C_{0}\right)
$$

$\alpha_{1}=\frac{\overline{\left(p^{\prime} d^{\prime}\right)_{s}} / \bar{\rho}}{2 \varepsilon_{s}}+\alpha_{1}^{i n c}$

As pointed out in detail in Pope (1994), the class of linear Reynolds stress closure for the slow part of the pressure strain correlation is used to obtain

$\phi_{i j}^{*}=\phi_{i j}-\frac{2}{3} \overline{p^{\prime} d^{\prime}} \delta_{i j}=\bar{\rho} \varepsilon_{s} A^{1} b_{i j}$

where $A^{1}$ is a model constant, thus corresponds to

$A^{1}=4 \alpha_{1}$

Using Eq. (33), we obtain

$A^{1}=4 \frac{\overline{\left(p^{\prime} d^{\prime}\right)_{s}} / \bar{\rho}}{2 \varepsilon_{s}}+A^{1 \text { inc }}$

$A^{1 i n c}=4 \alpha_{1}^{i n c}$

In Pope (1994), the coefficient $A^{1 \text { inc }}$ corresponds to Rotta model (1951)

$A^{1 \text { inc }}=-C_{1}$

Thus, we can obtain

$\phi_{i j}^{*}=-\left(C_{1}-2 \frac{\overline{\left(p^{\prime} d^{\prime}\right)_{s}} / \bar{\rho}}{2 \varepsilon_{s}}\right) \bar{\rho} \varepsilon_{s} b_{i j}$

In general, according to Sarkar et al. (1991) and Ristorcelli (1997), $\left(\overline{p^{\prime} d^{\prime}}\right)_{s}$ is proportional to $M_{t}^{2}$ as

$\left(\overline{p^{\prime} d^{\prime}}\right)_{s}=\beta \bar{\rho} M_{t}^{2} \varepsilon_{s}$

and finally, the compressibility modification model is proposed, namely :

$$
\phi_{i j}^{*}=-\left(C_{1}-b M_{t}^{2}\right) \bar{\rho} \varepsilon_{s} b_{i j}
$$

The coefficient $b$ is a model constant.

The revised models SSGa and SSGp using the compressibility correction in Eq. (40) are referred to SSGac and SSGpc respectively.

\section{Simulation Of Compressible HOMOGENEOUS SHEAR FLOW}

For homogeneous shear flow, the mean velocity gradient is given

$\tilde{U}_{i, j}=S \delta_{i 1} \delta_{j 2}$

where $S$ is the mean shear rate. Thus, the mean dilatation is

$\tilde{U}_{i, i}=0$

$\bar{\rho}=c t e$

The Favre averaged Reynolds stress should be solutions of the transport equation

Thus we can write 
$\bar{\rho} \frac{d}{d t}\left(R_{i j}\right)=P_{i j}+\phi_{i j}^{*}-\frac{2}{3} \bar{\rho} \varepsilon \delta_{i j}+\frac{2}{3} \overline{p^{\prime} d^{\prime}} \delta_{i j}$

The Favre averaged turbulent kinetic energy $K=R_{i i} / 2$ and its dissipation rate is obtained by solving the following transport equations:

$\bar{\rho} \frac{d}{d t} K=P-\bar{\rho} \varepsilon+\overline{p^{\prime} d^{\prime}}$

$\bar{\rho} \frac{d}{d t} \varepsilon_{s}=C_{\varepsilon 1} \bar{\rho} \frac{\varepsilon_{s}}{K} R_{k m} \frac{\partial}{\partial x_{m}} \tilde{U}_{k}$

$$
-C_{\varepsilon 2} \bar{\rho} \frac{\varepsilon_{s}^{2}}{K}
$$

where $C_{\varepsilon 1}=1.4$ and $C_{\varepsilon 2}=1.9$.

The turbulent Mach number is described by the transport equation as follow (see Speziale et al. 1995).

$$
\begin{gathered}
\frac{d}{d t} M_{t}=\frac{M_{t}}{2 \bar{\rho} K}\left(1+0.5 \gamma(\gamma-1) M_{t}^{2}\right) \\
\left(\overline{p^{\prime} d^{\prime}}-\bar{\rho} \varepsilon\right)+\frac{M_{t}}{2 K} P
\end{gathered}
$$

where $P=-\bar{\rho} R_{i j} \tilde{U}_{i, j}$ is the turbulent production and $\gamma=\frac{c_{p}}{c_{v}}$.

\section{RESULTS AND DISCUSSION}

The transport Eqs. (44), (45), (46) and (47) on which the second order closure for compressible homogeneous shear flow is based, are solved using the fourth-order accurate Runge-Kutta numerical scheme. The calibration of the coefficient $b$ in Eq. (40) based on the DNS results of Sarkar (1995) gives $b=1.6$ and the coefficient $\alpha=1.5$ in Eqs. (21) and (22).

Table 2 Initial conditions for the DNS results of Sarkar (1995) of compressible homogeneous shear

\begin{tabular}{|c|c|c|c|c|}
\hline case & $A_{1}$ & $A_{2}$ & $A_{3}$ & $A_{4}$ \\
\hline$M_{t 0}$ & 0.4 & 0.4 & 0.4 & 0.4 \\
\hline$\left(S K / \varepsilon_{s}\right)_{0}$ & 1.8 & 3.6 & 5.4 & 10.8 \\
\hline$M_{g 0}$ & 0.22 & 0.44 & 0.66 & 1.32 \\
\hline$b_{11}$ & 0 & 0 & 0 & 0 \\
\hline$b_{22}$ & 0 & 0 & 0 & 0 \\
\hline$b_{12}$ & 0 & 0 & 0 & 0 \\
\hline
\end{tabular}

Turbulence models for the dilatational part of the turbulent dissipation and the correlation pressure- dilatation are needed. For these terms, we choose the models proposed by Sarkar et al. (1991), namely:

$\varepsilon_{c}=0.5 M_{t}^{2} \varepsilon_{s}$

and

$$
\begin{aligned}
\overline{p^{\prime} d^{\prime}}= & 0.15 M_{t} \bar{\rho}\left(R_{i j}-\frac{2}{3} K \delta_{i j}\right) \\
& +0.2 \bar{\rho} M_{t}^{2} \varepsilon_{s}
\end{aligned}
$$

The ability of the proposed model to predict the anisotropy of compressible homogeneous turbulent shear flow will now be considered. The model predictions will be compared with DNS results developed by Sarkar (1995) for cases: $A_{1}, A_{2}, A_{3}$ and $\mathrm{A}_{4}$. These cases correspond to different initial conditions listed in Table 2. From all of the figures, it is clear that the incompressible SSG model (1991) referred to SSGi is still unable to predict the dramatic changes in the magnitude of the Reynoldsstress anisotropy that arise from compressibility. The proposed extension of the SSG model involves the turbulent Mach number. It provides an acceptable performance in compressible homogeneous shear flow. Figs. 1 to 12 show that the present model appears to be able to predict correctly the significant decrease in the magnitude of the normalized production term $-2 b_{12}$ and the increase in the magnitude of the streamwise $b_{11}$ and the transverse $b_{22}$ Reynolds-stress anisotropy. The proposed model yields reasonably acceptable results that are in good qualitative agreement with the DNS results of Sarkar (1995). An acceptable improvement of the results especially at high gradient Mach number $M_{g}=S \ell / \bar{a}$, where $\ell$ is an integral length scale, (see Sarkar 1995) and at high turbulent Mach number (cases $\mathrm{A}_{3}$ and $\mathrm{A}_{4}$ ) can be noticed with the use SSGac and SSGpc modified models.

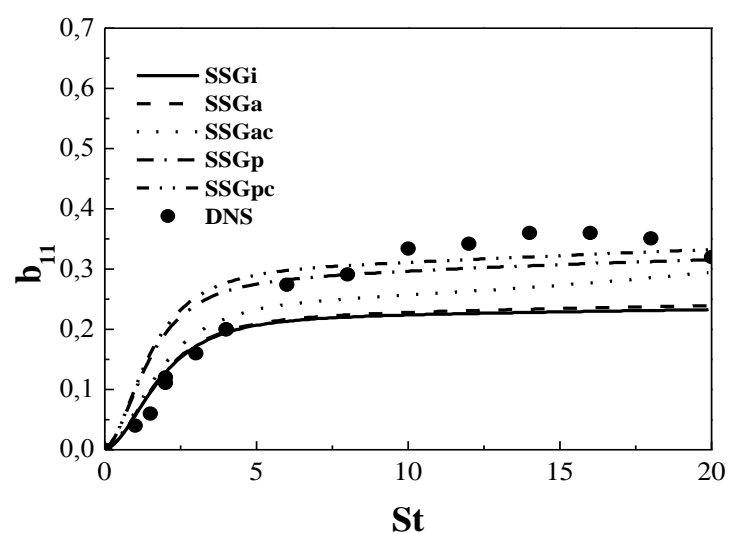

Fig. 1. Time evolution of the streamwise Reynolds stress anisotropy $\mathrm{b}_{11}$ in the case: $\mathrm{A}_{1}$ 


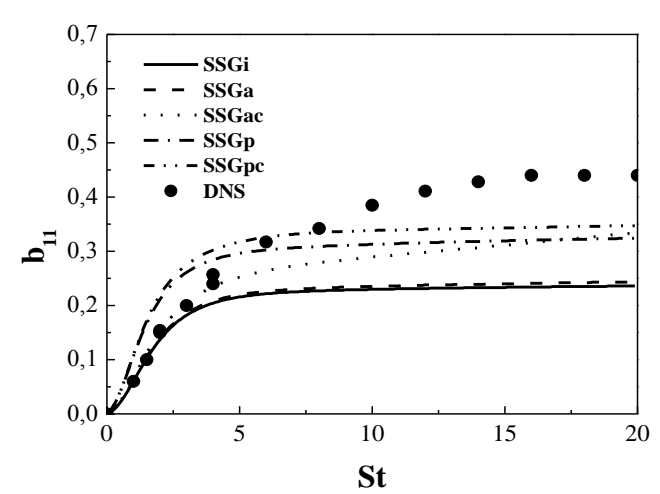

Fig. 2. Time evolution of the streamwise Reynolds stress anisotropy $b_{11}$ in the case: $A_{2}$

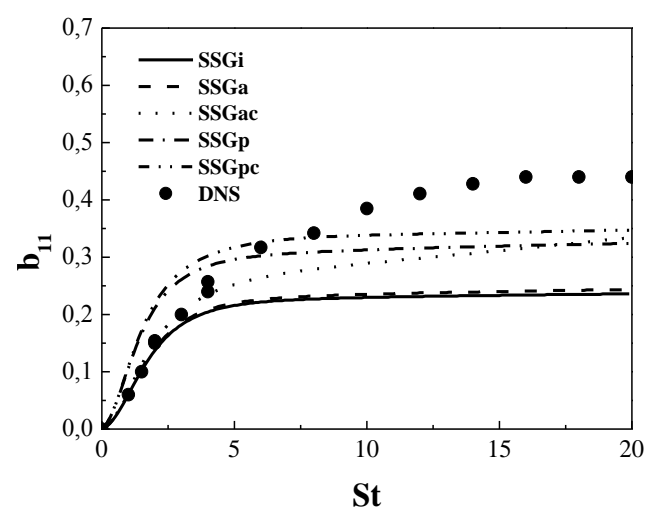

Fig. 3. Time evolution of the streamwise Reynolds stress anisotropy $\mathrm{b}_{11}$ in the case: $\mathrm{A}_{3}$

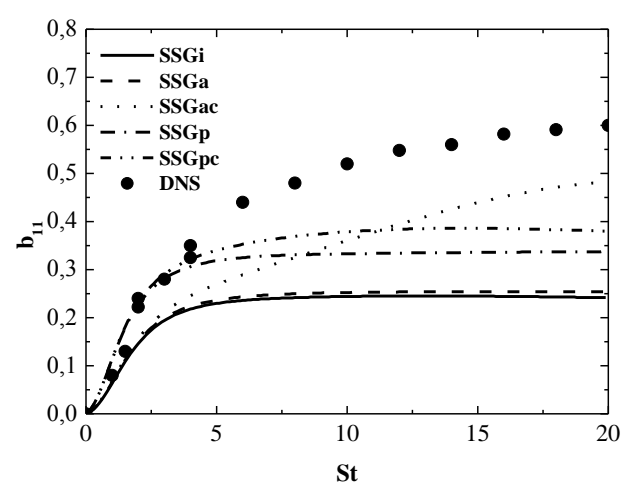

Fig. 4. Time evolution of the streamwise Reynolds stress anisotropy $b_{11}$ in the case: $A_{4}$

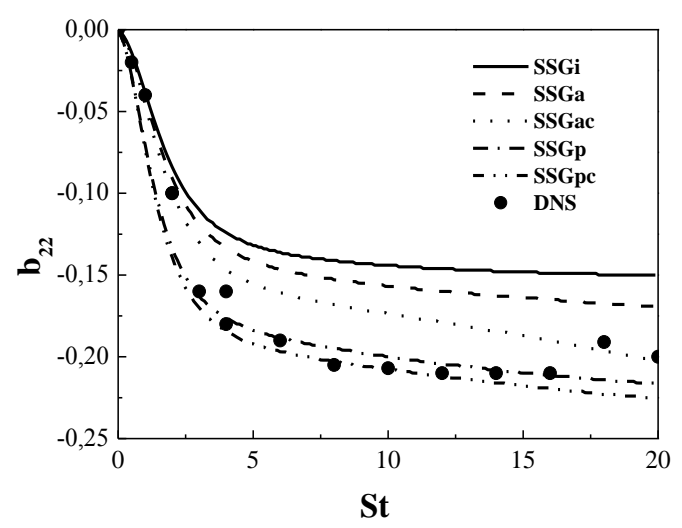

Fig. 5. Time evolution of the transverse Reynolds stress anisotropy $\mathrm{b}_{22}$ in the case: $\mathrm{A}_{1}$

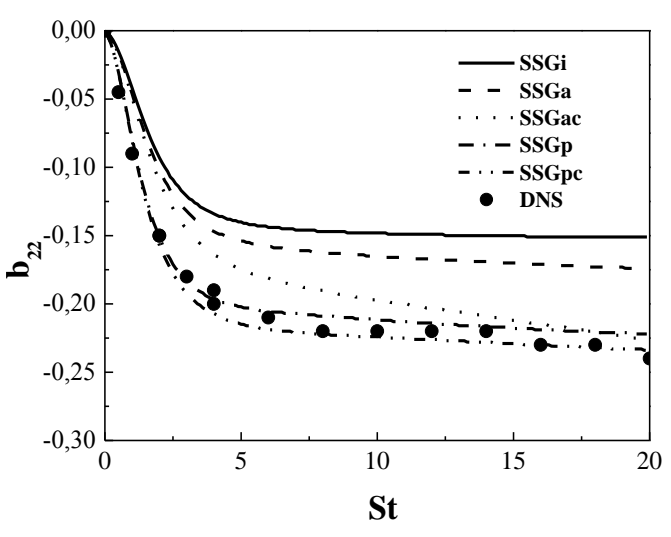

Fig. 6. Time evolution of the transverse Reynolds stress anisotropy $\mathrm{b}_{22}$ in the case: $\mathrm{A}_{2}$

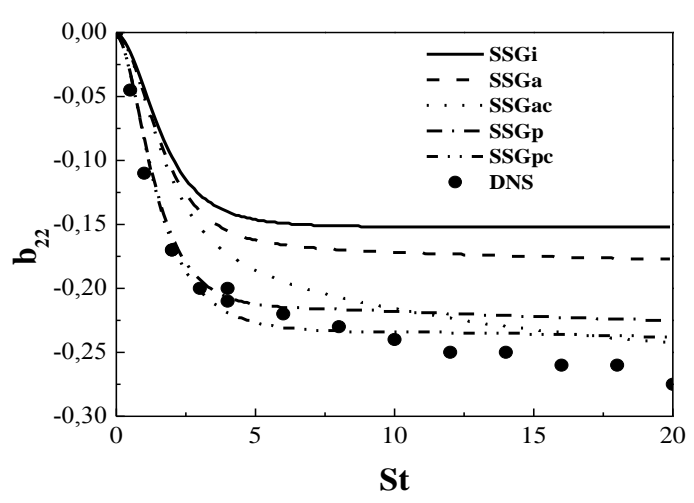

Fig. 7. Time evolution of the transverse Reynolds stress anisotropy $\mathrm{b}_{22}$ in the case: $\mathrm{A}_{3}$

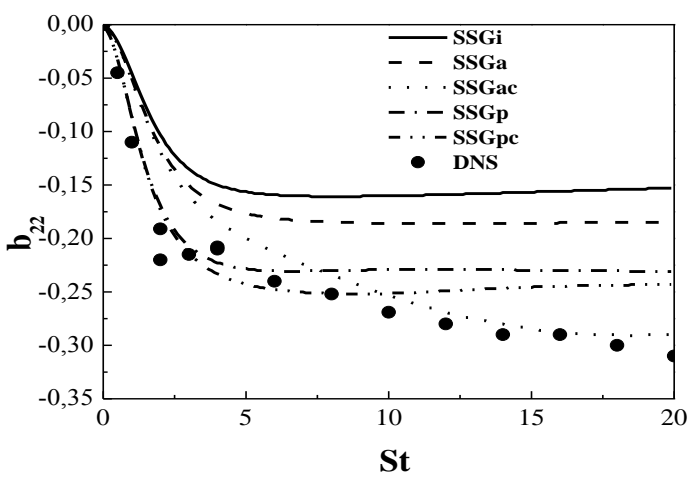

Fig. 8. Time evolution of the transverse Reynolds stress anisotropy $\mathrm{b}_{22}$ in the case: $\mathrm{A}_{4}$

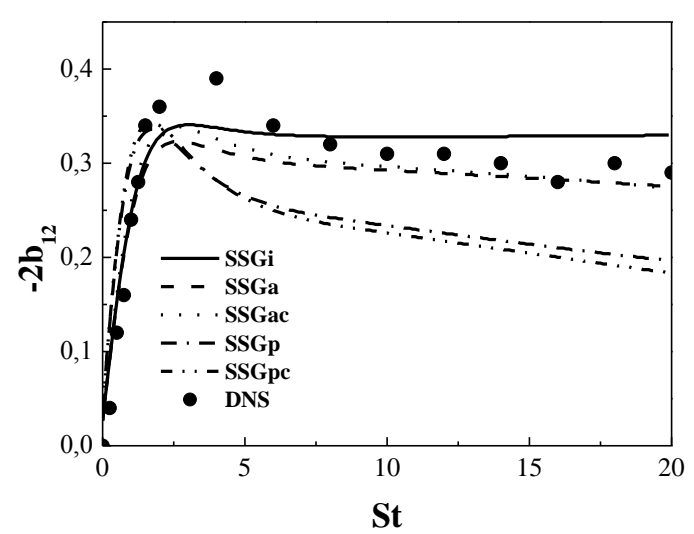

Fig. 9. Time evolution of the shear Reynolds stress anisotropy $\mathrm{b}_{12}$ in the case: $\mathrm{A}_{1}$ 


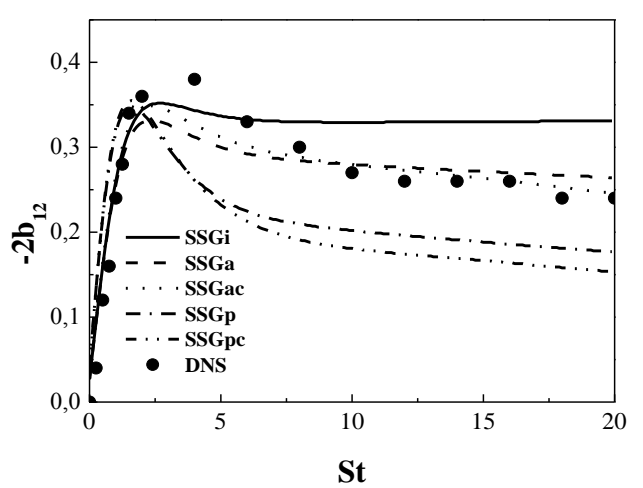

Fig. 10. Time evolution of the shear Reynolds stress anisotropy $\mathrm{b}_{12}$ in the case: $\mathrm{A}_{2}$

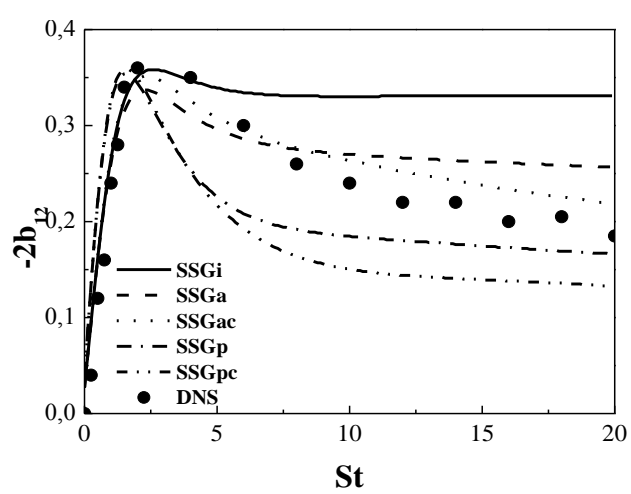

Fig. 11. Time evolution of the shear Reynolds stress anisotropy $b_{12}$ in the case: $A_{3}$

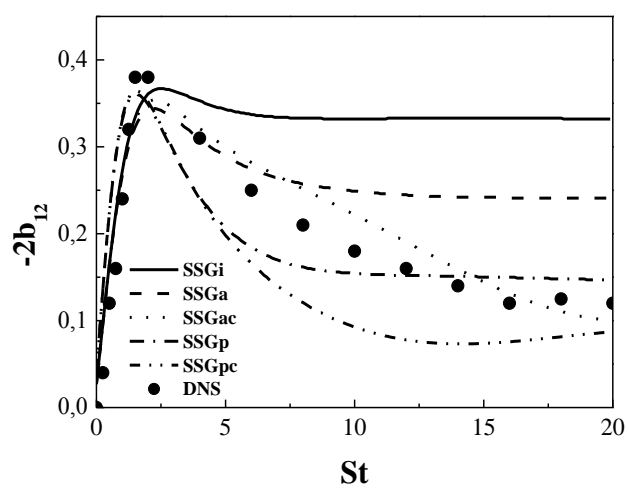

Fig. 12. Time evolution of the shear Reynolds stress anisotropy $\mathrm{b}_{12}$ in the case: $\mathrm{A}_{4}$

Figs. 13, 14, 15 and 16 present the behavior of the normalized dissipation $\left(\varepsilon_{s} / S K=-2 b_{12} \varepsilon_{s} / P\right)$, $\varepsilon_{s} / S K$ for cases: $\mathrm{A}_{1}, \mathrm{~A}_{2}, \mathrm{~A}_{3}$ and $\mathrm{A}_{4}$. It can be seen that there is a decrease in $\varepsilon_{s} / S K$ when $M_{g 0}$ increases, since the compressibility effects cause significant reduction in the Reynolds turbulent shear stress $b_{12}$ from numerical simulation cases: $\mathrm{A}_{1}$ to $\mathrm{A}_{4}$ of the previous DNS results. It is clear that the proposed model is in accordance with the DNS results. The predicted growth rates of the turbulent kinetic energy $\Lambda,(\Lambda=(d K / d t) / S K)$ with and without the compressibility corrections SSG model are potted in Figs. 17, 18, 19 and 20. It is clearly seen that all of the models appear to be able to predict accurately the trend of reduced growth rate with increasing the initial values of the gradient Mach number. This phenomenon has often been observed in DNS results of compressible homogeneous shear flow. The initial time values of $\Lambda$ show a systematic increase from cases $A_{1}$ to $A_{4}$. To find the causes of this discrepancy, an equation for $\Lambda$ obtained from Eq. (45) to be written as follows:

$\Lambda=-2 b_{12}(1-\chi)$

where $\chi=\frac{\varepsilon_{s}+\varepsilon_{c}-\overline{p^{\prime} d^{\prime}}}{S K}$ includes dilatational effects.

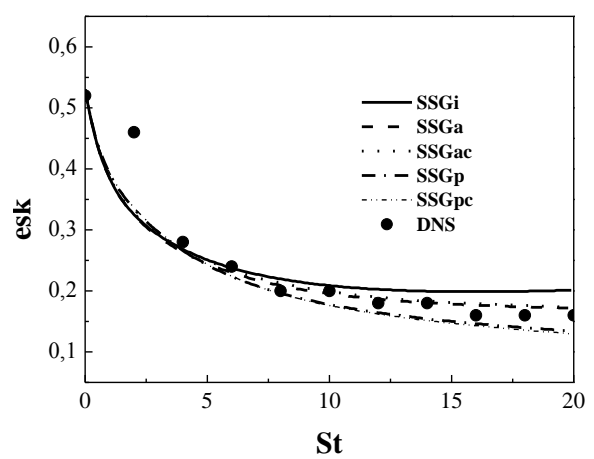

Fig. 13. Time evolution of the normalized dissipation $\left(\mathrm{esk}=\varepsilon_{s} / S K\right)$ in the case: $\mathrm{A}_{1}$

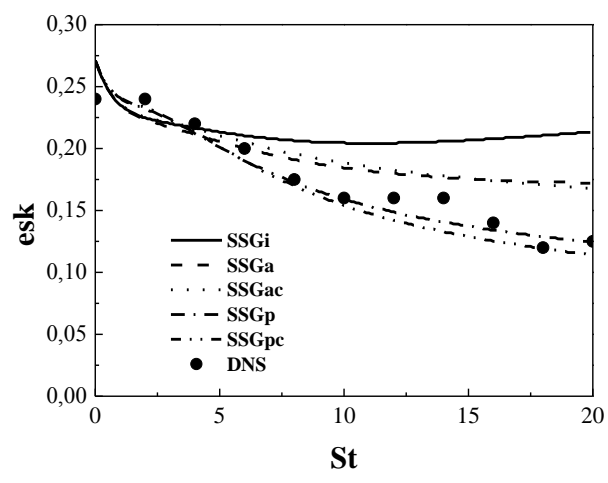

Fig. 14. Time evolution of the normalized dissipation $\left(\mathrm{esk}=\varepsilon_{s} / S K\right)$ in the case: $\mathrm{A}_{2}$

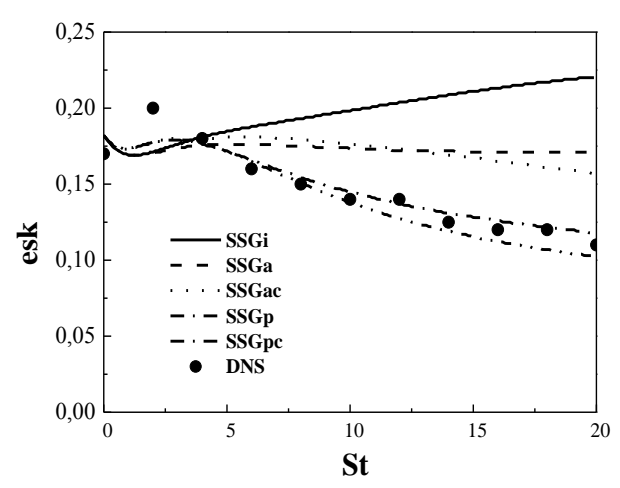

Fig. 15. Time evolution of the normalized dissipation $\left(\mathrm{esk}=\varepsilon_{s} / S K\right)$ in the case: $\mathrm{A}_{3}$ 


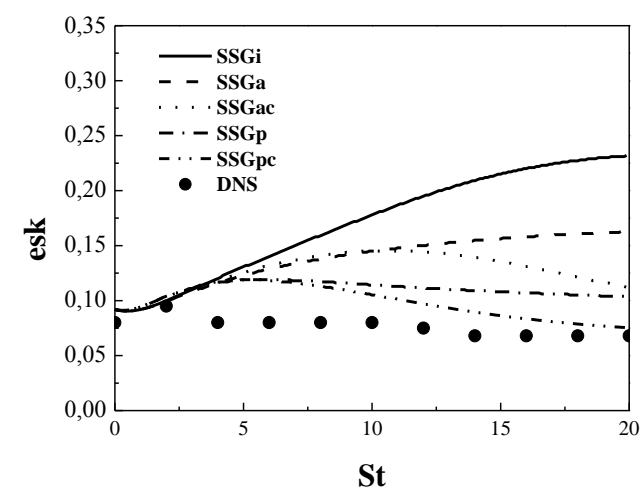

Fig. 16. Time evolution of the normalized dissipation $\left(\mathrm{esk}=\varepsilon_{s} / S K\right)$ in the case: $\mathrm{A}_{4}$

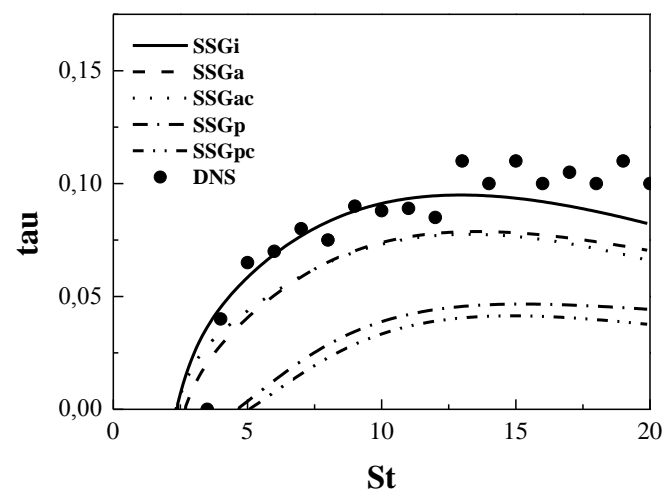

Fig. 17. Time evolution of the growth rate of the turbulent kinetic energy $(\operatorname{tau}=\Lambda)$ in the case: $A_{1}$

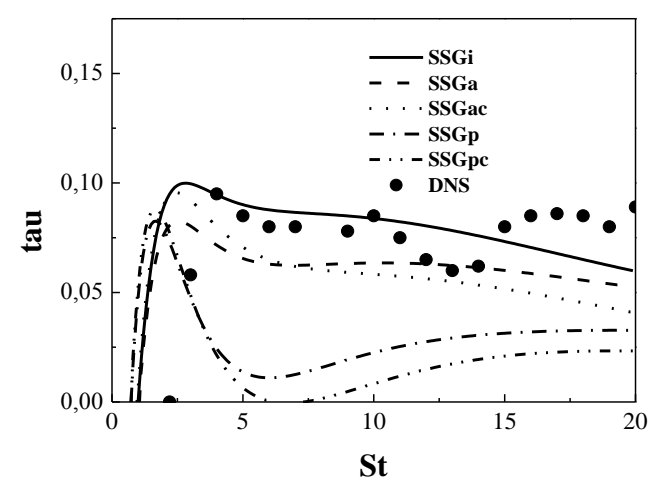

Fig. 18. Time evolution of the growth rate of the turbulent kinetic energy $(\operatorname{tau}=\Lambda)$ in the case: $A_{2}$

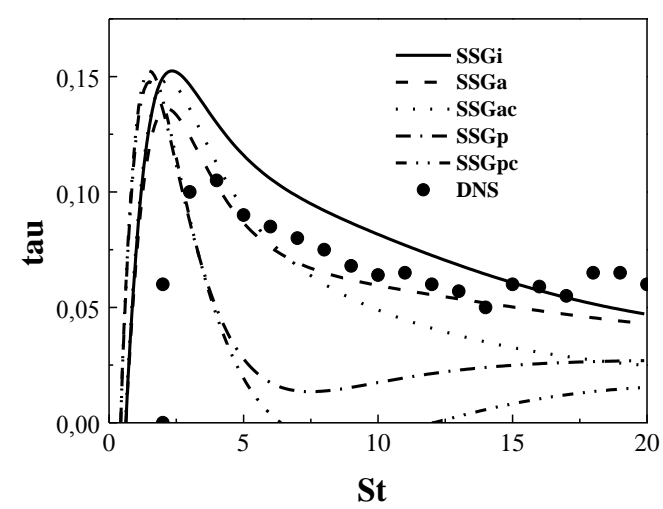

Fig. 19. Time evolution of the growth rate of the turbulent kinetic energy $(\operatorname{tau}=\Lambda)$ in the case: $\mathrm{A}_{3}$

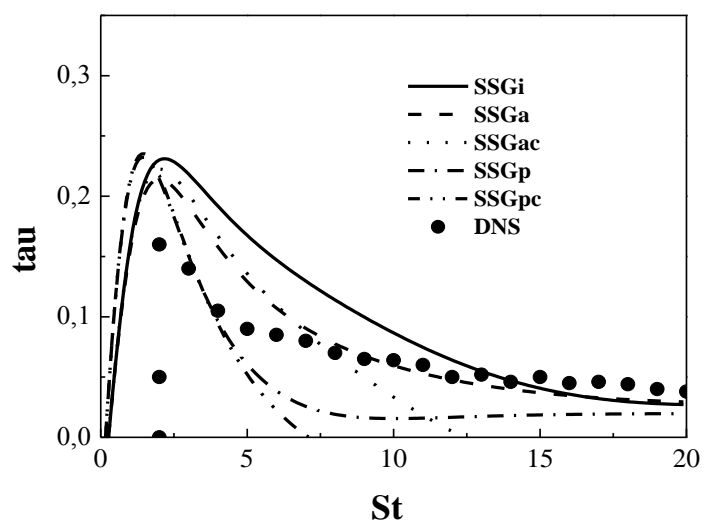

Fig. 20. Time evolution of the growth rate of the turbulent kinetic energy $(\operatorname{tau}=\Lambda)$ in the case: $\mathrm{A}_{4}$

Figs. 9, 10, 11 and 12 show that $-2 b_{12}$ seems to grow to constant maximum value which is about 0.38 for initial times $(S t \leq 4)$ and it shows very little differences among the different cases. According to the DNS results of Sarkar (1995), it is easy to see that the difference of $-2 b_{12}$ is much smaller than the difference of $\chi$ for initial times among cases $\mathrm{A}_{1}$ to $\mathrm{A}_{4}$. Also, the magnitude of the dilatational term: $\chi_{d}=\frac{\varepsilon_{c}-\overline{p^{\prime} d^{\prime}}}{S K}$ in case $\mathrm{A}_{4}$ is very larger relative to that in case $A_{1}$. This implies that the important increase in magnitude of $\chi_{d}$ is responsible for the increase in the initial time values of $\Lambda$ with increasing compressibility effects. On the contrary, for $(S t \geq 15), \quad \chi_{d} \quad$ becomes approximately very smaller and the reduced level of production is solely responsible for the reduced growth rate of turbulent kinetic energy.

In all of the figures, it is shown that the compressibility corrected models SSGa and SSGp yield considerably different predictions for the major structural compressibility parameters characteristic of flow. As it is indicated above, Adumitroiae et al. and Park et al. developed two different models for the pressure strain correlation to simulate compressible turbulent flows. In their approaches modeling, the part of pressure strain responsible to return to isotropy is simply defined as in incompressible model of Launder Reece and Rodi (1975). However, the remainder part which is called the rapid part (predictable in principle by the rapid distortion theory) is modified to become dependent on a turbulent Mach turbulent. From Figs. 1, 2, 3 and 4, one can remark that there is substantial differences between the two models SSGa and SSGp in their predictions, particularly for the anisotropy tensor $b_{11}$ for initial times $(S t \leq 4)$. This disparity in the initial time predictions of the models SSGa and SSGp arises from the way in which the rapid pressure strain is modeled. Also, the differences between the models can be seen in Figs. 9, 10, 11 and 12 that show the models predictions of the shear stress anisotropy $b_{12}$. It should be noted that the SSGa model yields a large 
improvement of the results for low gradient Mach number (cases: $\mathrm{A}_{1}$ and $\mathrm{A}_{4}$ ). The SSGp model shows an underestimation of the DNS results at these low gradient Mach numbers. Whereas, at high gradient Mach number (cases: $\mathrm{A}_{3}$ and $\mathrm{A}_{4}$ ), the SSGp model has better behavior. On the contrary, the SSGa model overestimates the DNS results for these ranges of gradient Mach number. As mentioned earlier, the difference in performance between SSGa and SSGp models is probably due to the fact that these models are derived by using two different approaches with an explicit account of the compressibility effects. In fact, the compressibility correction model by Adumitroiae et al(1999). is based on a procedure of the incompressible modeling that does not appear to be suited for high speed shear flow. While Park et al. use the concept of moving equilibrium in homogeneous turbulent shear flow to take into account the compressibility effects in the modeling of pressure-strain correlation. From the previous results, we can conclude that the Reynolds-stress closure using compressible SSG model involving the parameter $M_{t}$ appears to appropriate to intercept coherent compressibility effects on homogeneous turbulence at high speed shear flows.

\section{Conclusion}

In this study, the widely used second order closure has been used for the prediction of compressible homogeneous turbulent shear flow. The standard Reynolds-stress turbulence closure with the addition of the pressure-dilatation and compressible dissipation models yields very poor predictions of the changes in the Reynolds-stress anisotropy magnitude. The deficiency of this closure is due to the use of the incompressible models of the pressure-stress correlation. A new version of the extended SSG standard model has been proposed to reflect compressibility effects. Application of the model to predict compressible homogeneous shear flow shows satisfactory agreement with available DNS results. This model appears to be able to predict accurately the structural compressibility effects: the significant decrease in the magnitude of the Reynolds shear stress, the increase in the magnitude of diagonal components of the Reynolds-stress anisotropies and the reduction of the growth rate of the turbulent kinetic energy with increasing initial values of the gradient Mach number at high Mach number. The present model, successfully predicts the reduced of the normalized dissipation $\varepsilon_{s} / S K$. Therefore, the extension of the SSG model by using the turbulent Mach number is found out to be an important issue in the modeling of the pressure-strain correlation with respect to compressible turbulent flows.

\section{REFERENCES}

Adumitroiae, V., J.R. Ristorcelli and D.B. Taulbee (1999). Progress in Favre Reynolds stress closures for compressible flows. Phys. Fluids. A9, 2696-2719.
Hamba, F.(1999). Effects of pressure fluctuations on turbulence growth compressible homogeneous shear flow. Phys. Fluids. A11,1623-1625.

Launder, B. E., G.J. Reece and Rodi (1975). Progress in the development of a Reynolds stress turbulence closure. J. Fluid Mech. 68, 537-566.

Pantano. C. and S. Sarkar (2003). A study of compressibility effects in the high-speed turbulent shear layer using direct simulation. $J$. Fluid Mech. 451, 329-371.

Park, C.H. and S.O. Park (2005). Compressible turbulence model for the pressure-strain correlation. J. of Turbulence 6(2), 1-24.

Pope, S. B.(1994). On the relation between stochastic Lagrangian models of turbulence and second moment closures. Phys. Fluids. A2, 973-985.

Ristorcelli, J.R. (1997). A pseudo-sound constituve relationship for the dilatational covariances in compressible turbulence : An analytical theory. J .Fluid Mech. 347, 37-70.

Rotta, J. (1951). Statische theorie nichthomogener turbulenz. Zeitschrift für Physik 129, 547-572.

Sarkar,S., G. Erlebacher, M.Y. Hussaini and H.O. Kreiss (1991). The analysis and modeling of dilatational terms in compressible turbulence. $J$. Fluid Mech. 227, 473-493.

Sarkar, S. (1995). The stabilizing effects of compressibility in turbulent shear flow. J. Fluid Mech 282, 163-186.

Speziale, C.G., R. Abid and N.N. Mansour (1995). Evaluation of Reynolds stress turbulence closures in compressible homogeneous shear flow. J. Appl. Math. Phys. 46, 5717-5736.

Speziale,C.G.,S. Sarkar and T.B. Gatski (1991). Modeling the pressure-strain correlation of turbulence: an invariant dynamical systems approach. J. Fluid Mech.227,245-272. 
K. Hechmi et al. / JAFM, Vol. 5, No. 4, pp. 101-111, 2012.

Vreman,A.W., N.D. Sandham and K.H. Luo (1996). Compressible mixing layer growth rate and turbulence characteristics. J. Fluid Mech. $320,320-325$.

Younis, Y., A. Bibi, A. U. Haque and S. Khushnood (2209). Vortical flow topology on windward and leeward side of delta wing at supersonic speed, Journal of Applied Fluid Mechanics 2(4), 13-21.

Zeman, O. (1990). Dilatational dissipation, the concept and application in modeling compressible mixing layers. Phys. Fluids. A2, 178-188. 